Research Article

\title{
Faults Detection Using Sliding Mode Observer and Its Application on Elevating Servo Systems
}

\author{
Lingfei Tian $\left(\mathbb{D},{ }^{1}\right.$ Linfang Qian, ${ }^{1}$ Miao $\mathrm{Li}^{2}$ and Jiawei Fu $\mathbb{D}^{1}$ \\ ${ }^{1}$ School of Mechanical Engineering, Nanjing University of Science and Technology, Nanjing 210094, China \\ ${ }^{2}$ Norinco Group Planning and Research Institute, Beijing 100053, China \\ Correspondence should be addressed to Lingfei Tian; tlfnjust@163.com
}

Received 17 June 2019; Revised 15 August 2019; Accepted 6 September 2019; Published 7 October 2019

Academic Editor: Daniel Morinigo-Sotelo

Copyright (c) 2019 Lingfei Tian et al. This is an open access article distributed under the Creative Commons Attribution License, which permits unrestricted use, distribution, and reproduction in any medium, provided the original work is properly cited.

\begin{abstract}
The elevating servo system (ESS) of vehicle-mounted howitzer (VMH) is a typical closed-loop electrohydraulic position servo system, and the faults of its actuator and sensor seriously affect the safety and reliability of the system. In practice, model uncertainty, nonlinearities, unknown disturbance, and output noise present enormous challenges to conduct fault detection of the system. In the current paper, an online fault detection scheme using the sliding mode technology is proposed. Not only the derivation method of state equation and some common fault expressions but also a new design of sliding mode observer with the ability to eliminate the influences of the above factors on detection results is given. The observer's parameter matrices are obtained by the linear matrix inequality. To promote the fault detection capability, a statistical-based dynamic threshold is developed to detect actuator faults and sensor faults simultaneously. Finally, experimental studies are implemented on a test rig for validating the system model, and the results of four experiments show the effectiveness of proposed methods.
\end{abstract}

\section{Introduction}

Concerns on the safety and reliability of weapon equipment systems facilitate the development of fault detection techniques for complex industrial systems, which has been considered as a significant approach in pursuing a practical solution to guarantee the continuous and stable operation of the actual systems [1]. The ESS plays an important role in the pointing control of barrels, which is a typical closed-loop control system. To avoid the trend of serious performance degradation, the schemes proposed should be capable of preventing the dissemination of fault effects when possible failure of certain components happens.

Mostly, the ESS is working in the wild field with heavy electromagnetic interference (EMI) and environment temperature range of $-40^{\circ} \mathrm{C}$ to $50^{\circ} \mathrm{C}$. Model parameters perturbation and output disturbance are inevitable as the viscosity of hydraulic fluid varies with temperature, and sensor performances change due to EMI. The model linearization method has been studied in modeling electrohydraulic servo systems [2-4]. However, linearization errors between the linearized model and the actual nonlinear one must be compensated for better performance and accuracy. Knowledge-based fault diagnosis methods, such as neural network and support vector machine, have been employed in system modeling [5-8]. However, a large number of sample data needs to be provided in advance for training the neural network, which is impractical and inconvenient for the weapon equipment system with harsh trial conditions as well as insufficient trial times.

In recently decades, sliding mode variable structure technology has been widely applied in various fields for its suppression of external disturbances and insensitivity to bounded disturbances. Fault detection methods based on the sliding mode observer have been extensively studied. Li et al. [9] designed a sliding mode observer of a buck-boost converter and addressed the problem of sensor faults detection and estimation. Liu and Shi [10] proposed a sliding mode control scheme against sensor faults and disturbances 
simultaneously. Mao et al. [11] developed a sensor fault detection scheme for rail vehicle suspension in the presence of uncertainty, noises, and stochastic process signals. The methods mentioned above focus on sensor faults detection only, which omit actuator fault detection and have not been implemented on test rigs.

Model uncertainty, nonlinearity, and output stochastic noises are the main reasons for the difficult extension of fault detection technology in practice [12-15]. By taking the sensor fault vector as a part of an extended state vector and constructed an augmented singular system, Yang et al. [12] developed a robust sliding mode observer to detect actuator faults, in which only the actuator faults and unknown inputs are considered.

Faults in the system can be roughly divided into two categories: actuator faults and sensor faults. To the whole systems, the objective is not only to consider the nonlinear characteristics of the system and the working environment but also to establish a unified fault detection method for the two types of faults. The fault detection observer proposed in this work is partially motivated by the study in [12], employing a different structure with the unknown input, actuator, and sensor faults. Without loss of generality, the output noise is also considered in this paper for the enhancement of output disturbance under harsh environments. Firstly, the sensor faults and output noise are considered as a part of new states, and a new augmented system is reconstructed. Secondly, a fault detection observer is designed for this augmented system to detect sensor faults and actuator faults simultaneously. Finally, based on the experiment data, a dynamic threshold is developed to reduce the false alarm. Moreover, all the results are verified on an actual test rig.

The paper is organized as follows. Section 2 describes the experimental setup and system modeling and gives the derivation process of state equation and some common fault expressions. Section 3 presents a fault detection algorithm to the ESS, in which a sliding mode observer and a dynamical threshold are developed. In Section 4, the performance of the proposed method is demonstrated on the test rig. Conclusions are stated in Section 5.

\section{Experimental Setup and System Modeling}

2.1. Description of Experimental Test Rig. Figure 1 shows the test rig used in these experiments. The main hardware components of the elevating servo system of VMH are as follows: an elevating hydraulic cylinder, a proportional valve, a pair of hydraulic lock valve, an accumulator, a hydraulic pump station with a gear pump, a relief valve, and other accessories. The flow rate and operation pressure of the hydraulic pump station are $80 \mathrm{l} / \mathrm{min}$ and 120 bar, respectively. The proportional valve determines the flow rate controlling the extension and retraction of the piston rod of the elevating cylinder that changes the elevating angles of the barrel of the VMH. By comparing with the command signals, the controller gives a feedback signal and finally controls the barrel to reach the target

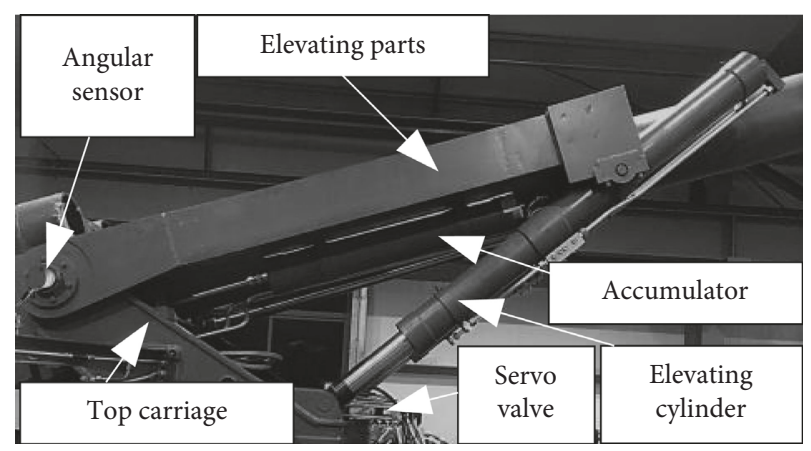

FIgURE 1: Experimental test rig of ESS.

elevating angle. In order to bear the load of the heavy barrel, the ordinary dual-acting elevating cylinder is designed with a third independent chamber. The chamber connected with the precharged accumulator through an independent pipeline is called the elevating-counterbalance cylinder.

Figure 2 is the diagram of ESS, where $\mathrm{O}$ defines the trunnion between the top carriage and elevating parts, $\mathrm{A}$ and $\mathrm{B}$ are the hinge joints between the elevating cylinder and the cradle and between the elevating cylinder and the top carriage, respectively, $\overline{O A}=b, \overline{O B}=a, p_{\mathrm{s}}$ and $p_{\mathrm{r}}$ are the proportional valve entrance port and return port pressures, respectively, $p_{1}$ and $p_{2}$ are the working chambers pressures, respectively, $p_{3}$ denotes the pressure of third independent chamber, $A_{1}$ and $A_{2}$ are effective areas of the two elevating cylinder chambers, respectively, $A_{3}$ is the area of third independent chamber, $\theta_{0}$ is the included angle between $\overline{O A}$ and $\overline{O B}$ while elevating hydraulic cylinder has the minimum displacement, $\theta$ is the rotation angle (elevating angle) of elevating parts around trunnion $O$ and $F_{\mathrm{c}}$ is the force applied on the cylinder by the elevating parts.

2.2. System Modeling. The valve spool's first-order dynamics is considered since the frequency response of the used proportional valve is far greater than the hydraulic actuator's. Thus,

$$
u=\frac{\tau}{k_{\mathrm{v}}} \dot{x}_{\mathrm{v}}+\frac{1}{k_{\mathrm{v}}} x_{\mathrm{v}},
$$

where $x_{\mathrm{v}}$ is the proportional valve spool displacement, $u$ is the control input, $\tau$ is the time constant, and $k_{\mathrm{v}}$ is the gain.

The flow rates through the proportional valve are as follows:

$$
\begin{array}{r}
Q_{1}= \begin{cases}k_{q}\left(x_{\mathrm{v}}-d_{\mathrm{v}}\right) \sqrt{p_{\mathrm{s}}-p_{1}}, & x_{\mathrm{v}}>d_{\mathrm{v}}, \\
0, & x_{\mathrm{v}} \leq\left|d_{\mathrm{v}}\right|, \\
k_{q}\left(x_{\mathrm{v}}+d_{\mathrm{v}}\right) \sqrt{p_{1}-p_{\mathrm{r}}}, & x_{\mathrm{v}}<-d_{\mathrm{v}},\end{cases} \\
Q_{2}= \begin{cases}k_{\mathrm{q}}\left(x_{\mathrm{v}}-d_{\mathrm{v}}\right) \sqrt{p_{2}-p_{\mathrm{r}}}, & x_{\mathrm{v}}>d_{\mathrm{v}}, \\
0, & x_{\mathrm{v}} \leq\left|d_{\mathrm{v}}\right|, \\
k_{\mathrm{q}}\left(x_{\mathrm{v}}+d_{\mathrm{v}}\right) \sqrt{p_{\mathrm{s}}-p_{2}}, & x_{\mathrm{v}}<-d_{\mathrm{v}},\end{cases}
\end{array}
$$




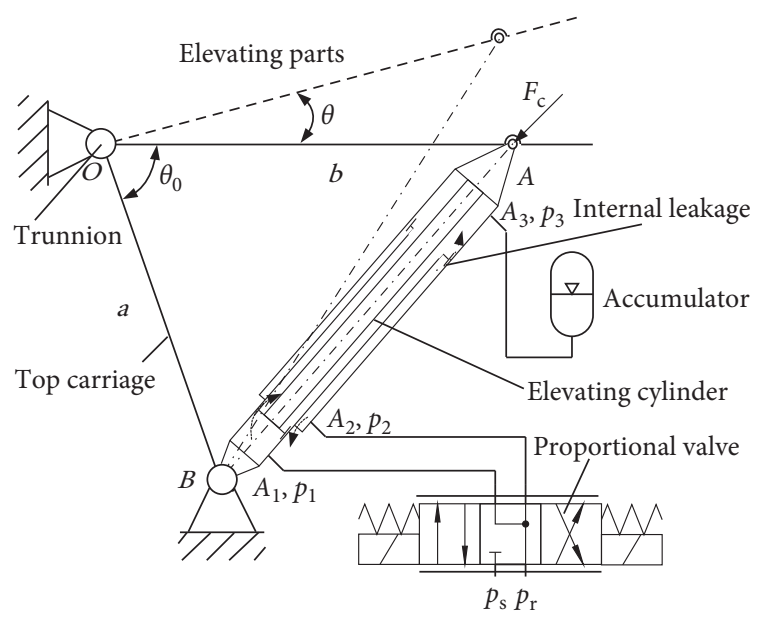

FIGURE 2: Diagram of ESS.

where $\left(x_{\mathrm{v}} \pm d_{\mathrm{v}}\right)$ is the orifice open size managed by the spool overlap $d_{\mathrm{v}}$. The flow rate coefficient $k_{\mathrm{q}}=C_{\mathrm{d}} \omega \sqrt{2 / \rho}, C_{\mathrm{d}}$ is the discharge coefficient, $\omega$ is the proportional valve area gradient, and $\rho$ is the fluid density.

Taking leakage and fluid compressibility into consideration, the flow continuity equation of the hydraulic cylinder can be written as

$$
\begin{aligned}
Q_{1} & =\frac{\left(V_{01}+A_{1} x_{\mathrm{p}}\right) \dot{p}_{1}}{\beta_{\mathrm{e}}+A_{1} \dot{x}_{\mathrm{p}}+C_{i}\left(p_{1}-p_{2}\right)}, \\
Q_{2} & =\frac{A_{2} \dot{x}_{\mathrm{p}}+C_{i}\left(p_{1}-p_{2}\right)-C_{\mathrm{e}} p_{2}-\left(V_{02}-A_{2} x_{\mathrm{p}}\right) \dot{p}_{2}}{\beta_{\mathrm{e}}},
\end{aligned}
$$

where $Q_{1}$ and $Q_{2}$ are flow into chamber without rod and chamber with rod, respectively, $V_{01}$ and $V_{02}$ are volume of extension chamber and retraction chamber, respectively, $x_{\mathrm{p}}$ denotes the displacement of the piston, $C_{\mathrm{i}}$ is the internal leakage coefficient, $C_{\mathrm{e}}$ is the external leakage coefficient of the cylinder, and $\beta_{\mathrm{e}}$ is the fluid effective bulk modulus.

According to Newton's second law, the equation describing the piston motion is given by

$$
p_{1} A_{1}-p_{2} A_{2}=m_{\mathrm{e}} \ddot{x}_{\mathrm{p}}+B_{\mathrm{p}} \dot{x}_{\mathrm{p}}+F_{\mathrm{f}}+F_{\mathrm{c}}-F,
$$

where $m_{\mathrm{e}}$ is the equivalent mass of the objects moving with the piston, $B_{\mathrm{p}}$ accounts for the effective viscous coefficient, $F_{\mathrm{f}}$ is the unknown friction, $F$ denotes the pressure of third independent chamber, and $F=p_{3} A_{3}$. Defining $F_{\mathrm{d}}=F_{\mathrm{f}}+F_{\mathrm{c}}$, equation (4) can be rewritten as

$$
p_{1} A_{1}-p_{2} A_{2}=m_{\mathrm{e}} \ddot{x}_{\mathrm{p}}+B_{\mathrm{p}} \dot{x}_{\mathrm{p}}-F+F_{\mathrm{d}} .
$$

Let the state variable $\mathbf{x}=\left[\begin{array}{llll}x_{1} & x_{2} & x_{3} & x_{4}\end{array}\right]^{\mathrm{T}}=$ $\left[\begin{array}{llll}\dot{x}_{\mathrm{p}} & p_{1} & p_{2} & x_{\mathrm{v}}\end{array}\right]^{\mathrm{T}}$. From equations (1)-(7), the state space model of the entire system can be expressed as

$$
\begin{aligned}
& \left\{\begin{array}{l}
\dot{\mathbf{x}}=\mathbf{A} \mathbf{x}+\mathbf{B} \boldsymbol{u}+\mathbf{g}(\mathbf{x})+\mathbf{D} F_{\mathrm{d}}, \\
\mathbf{y}=\mathbf{C} \mathbf{x}+\mathbf{E}_{\mathrm{d}} \mathbf{d},
\end{array}\right. \\
& \mathbf{A}=\left[\begin{array}{cccc}
-\frac{B_{p}}{m_{\mathrm{e}}} & \frac{A_{1}}{m_{\mathrm{e}}} & -\frac{A_{2}}{m_{\mathrm{e}}} & 0 \\
\frac{-A_{1} \beta_{\mathrm{e}}}{V_{01}} \frac{-C_{\mathrm{i}} \beta_{\mathrm{e}}}{V_{01}} & \frac{C_{\mathrm{i}} \beta_{\mathrm{e}}}{V_{01}} & 0 \\
\frac{A_{2} \beta_{\mathrm{e}}}{V_{02}} & \frac{C_{\mathrm{i}} \beta_{\mathrm{e}}}{V_{02}} & \frac{-\left(C_{\mathrm{i}}+C_{\mathrm{e}}\right) \beta_{\mathrm{e}}}{V_{02}} & 0 \\
0 & 0 & 0 & -\frac{1}{\tau}
\end{array}\right] ;
\end{aligned}
$$

$\mathbf{B}=\left[\begin{array}{c}0 \\ 0 \\ 0 \\ \frac{k_{\mathrm{v}}}{\tau}\end{array}\right] ;$

$\mathbf{C}=\left[\begin{array}{cccc}10^{3} & 0 & 0 & 0 \\ 0 & 10^{-6} & 0 & 0 \\ 0 & 0 & 10^{-6} & 0 \\ 0 & 0 & 0 & 10^{3}\end{array}\right]$;

$\mathbf{g}(\mathbf{x})=\left[\begin{array}{c}\frac{F}{m_{\mathrm{e}}} \\ \frac{\mathbf{g}_{1}(\mathbf{x}) \beta_{\mathrm{e}}}{V_{01}} \\ -\frac{\mathbf{g}_{2}(\mathbf{x}) \beta_{\mathrm{e}}}{V_{02}} \\ 0\end{array}\right]$,

$\mathbf{g}_{1}(\mathbf{x})= \begin{cases}k_{\mathrm{q}}\left(x_{4}-d_{\mathrm{v}}\right) \sqrt{p_{\mathrm{s}}-x_{2}}, & x_{\mathrm{v}}>d_{\mathrm{v}}, \\ k_{\mathrm{q}}\left(x_{4}+d_{\mathrm{v}}\right) \sqrt{x_{2}-p_{\mathrm{r}}}, & x_{\mathrm{v}}<-d_{\mathrm{v}},\end{cases}$

$\mathbf{g}_{2}(\mathbf{x})= \begin{cases}k_{\mathrm{q}}\left(x_{4}-d_{\mathrm{v}}\right) \sqrt{x_{3}-p_{\mathrm{r}}}, & x_{\mathrm{v}}>d_{\mathrm{v}}, \\ k_{\mathrm{q}}\left(x_{4}+d_{\mathrm{v}}\right) \sqrt{p_{\mathrm{s}}-x_{3}}, & x_{\mathrm{v}}<-d_{\mathrm{v}},\end{cases}$ 
where $\mathbf{x} \in R^{4 \times 1}$ is the state variable, $\mathbf{y} \in R^{4 \times 1}$ is the output vector, $\boldsymbol{E}_{\mathrm{d}}$ is the known disturbance distribution matrix, $\mathbf{d}$ is the output disturbance or measurement noise vector (Gauss white noise included in the brief), and its derivatives $\dot{d}$ and $L_{2}$ norms are bounded. There is a geometrically one-to-one correspondence between elevating angle $\theta$ measured by an angular sensor and the piston displacement. The piston velocity can be obtained by the derivative of the piston displacement $x_{\mathrm{p}}$. Spool displacement sensor is embedded in proportional valve.

2.3. Fault Analysis and Modeling. The high-frequency faults occurred during the operation of ESS are system pressure, cylinder leakage, proportional valve amplifier drift, and sensor drift. The relevant state variables and the model parameters included in the state equation will change when faults occur. Defining the matrix deviation of the parameter matrix $\mathbf{A}, \mathbf{B}$, and $\mathbf{g}(\mathbf{x})$ in the state equation as $\Delta \mathbf{A}, \Delta \mathbf{B}$, and $\Delta \mathbf{g}(\mathbf{x})$, respectively:

$$
\begin{aligned}
& \Delta \mathbf{A}=\left[\begin{array}{cccc}
0 & 0 & 0 & 0 \\
0 \frac{-\Delta C_{i} \beta_{e}}{V_{01}} & \frac{\Delta C_{i} \beta_{e}}{V_{01}} & 0 \\
0 & \frac{\Delta C_{i} \beta_{e}}{V_{02}} \frac{-\left(\Delta C_{i}+\Delta C_{e}\right) \beta_{e}}{V_{02}} & 0 \\
0 & 0 & 0 & 0
\end{array}\right], \\
& \Delta \mathbf{B}=\left[\begin{array}{llll}
0 & 0 & 0 & \frac{\Delta k_{v}}{\tau}
\end{array}\right]^{\mathrm{T}},
\end{aligned}
$$

$$
\Delta \mathbf{g}(\mathbf{x})=\left[\begin{array}{c}
0 \\
\frac{\Delta \mathbf{g}_{1}(\mathbf{x}) \beta_{e}}{V_{01}} \\
-\frac{\Delta \mathbf{g}_{2}(\mathbf{x}) \beta_{e}}{V_{02}} \\
0
\end{array}\right] .
$$
follows:

Three types faults $\mathbf{f}_{a}^{i}(i=1,2,3)$ are considered as

$$
\begin{aligned}
& f_{a}^{1}=\left\{\begin{array}{l}
f_{a 1}^{1}, \\
f_{a 2}^{1},
\end{array}\right. \\
& f_{\mathrm{a}}^{2}= \begin{cases}\frac{f_{\mathrm{a} 1}^{2}=-\beta_{e} \Delta C_{\mathrm{i}}\left(x_{2}-x_{3}\right)}{V_{01}}, & x_{v}>d_{v}, \\
\frac{f_{\mathrm{a} 2}^{2}=\beta_{e} \Delta C_{\mathrm{i}}\left(x_{2}-x_{3}\right)}{V_{02}}-\frac{\beta_{e} \Delta C_{e} x_{3}}{V_{02}}, & x_{v}<-d_{v},\end{cases} \\
& f_{\mathrm{a}}^{3}=\frac{\Delta k_{v}}{\tau} u,
\end{aligned}
$$

where

$$
\begin{aligned}
& f_{a 1}^{1}= \begin{cases}\left(k_{q} \Delta x_{4} \sqrt{p_{s}-x_{2}}+k_{q}\left(x_{4}-d_{v}\right) \frac{\Delta p_{s}-\Delta x_{2}}{2 \sqrt{p_{s}-x_{2}}}\right) \frac{\beta_{e}}{V_{01}}, & x_{v}>d_{v}, \\
\left(k_{\mathrm{q}} \Delta x_{4} \sqrt{x_{2}-p_{r}}+k_{\mathrm{q}}\left(x_{4}+d_{v}\right) \frac{\Delta x_{2}}{2 \sqrt{x_{2}-p_{r}}}\right) \frac{\beta_{e}}{V_{01}}, & x_{v}<-d_{v},\end{cases} \\
& f_{a 2}^{1}= \begin{cases}-\left(k_{q} \Delta x_{4} \sqrt{x_{3}-p_{r}}+k_{q}\left(x_{4}-d_{\mathrm{v}}\right) \frac{\Delta x_{3}}{2 \sqrt{x_{3}-p_{r}}}\right) \frac{\beta_{e}}{V_{02}}, & x_{v}>d_{v}, \\
-\left(k_{q} \Delta x_{4} \sqrt{p_{s}-x_{3}}+k_{q}\left(x_{4}+d_{v}\right) \frac{\Delta p_{s}-\Delta x_{3}}{2 \sqrt{p_{s}-x_{3}}}\right) \frac{\beta_{e}}{V_{02}}, & x_{v}<-d_{v} .\end{cases}
\end{aligned}
$$

where $\Delta C_{\mathrm{i}}, \Delta C_{e}$, and $\Delta p_{s}$ are the deviations of internal leakage coefficient, external leakage coefficient, and supply pressure, respectively, $\Delta x_{2}, \Delta x_{3}$, and $\Delta x_{4}$ are the changes of system state vector component, and $\Delta \dot{x}_{\mathrm{p}}$ is the sensor deviation. Return pressure $p_{\mathrm{r}}$ is regarded as a small positive constant because of low pressure. Thus, the above faults, equations (9) (14), can be expressed by

$$
\begin{aligned}
\mathbf{F}_{a} & =\left[\begin{array}{lll}
0 & 0 & 0 \\
1 & 0 & 0 \\
0 & 1 & 0 \\
0 & 0 & 1
\end{array}\right], \\
\mathbf{f}_{\mathrm{a}} & =\left[\begin{array}{lll}
f_{\mathrm{a} 1}^{1}+f_{\mathrm{a} 1}^{2} & f_{\mathrm{a} 2}^{1}+f_{\mathrm{a} 2}^{2} & f_{\mathrm{a}}^{3}
\end{array}\right]^{\mathrm{T}} .
\end{aligned}
$$


The entire state space equation with the faults can be written as

$$
\left\{\begin{array}{l}
\dot{\mathbf{x}}=\mathbf{A x}+\mathbf{B} u+\mathbf{g}(\mathbf{x})+\mathbf{D} F_{\mathrm{d}}+\mathbf{F}_{\mathrm{a}} \mathbf{f}_{\mathrm{a}} \\
\mathbf{y}=\mathbf{C} \mathbf{x}+\mathbf{E}_{d} \mathbf{d}+\mathbf{F}_{\mathrm{s}} \mathbf{f}_{s}
\end{array}\right.
$$

where $\mathbf{F}_{a} \mathbf{f}_{a}=\Delta \mathbf{A x}+\Delta \mathbf{B} u+\Delta \mathbf{g}(\mathbf{x})$ and $\mathbf{F}_{a} \in R^{4 \times 3}$ is the actuator fault distribution matrix. Assume that the actuator fault vector $\mathbf{f}_{a} \in R^{3 \times 1}$ satisfies $\left\|\mathbf{f}_{a}\right\| \leq \alpha_{M}$, where $\alpha_{M}$ is a known real constant. The $i$-th fault is expressed as $\mathbf{f}_{a}^{i}(i=1,2,3)$. Unknown disturbance $\mathbf{F}_{\mathrm{d}}$ satisfies $\left\|F_{0}\right\| \leq \beta_{M}$. $\mathbf{F}_{\mathrm{s}} \in R^{4 \times 1}$ is the sensor fault distribution matrix, and $\mathbf{F}_{\mathrm{s}}=\left[\begin{array}{llll}1 & 0 & 0 & 0\end{array}\right]^{\mathrm{T}} . \mathbf{f}_{s} \in R^{1}$ is the sensor fault vector, and the $i$-th fault is expressed as $\mathbf{f}_{s}^{i}(i=1,2, \ldots) \mathbf{f}_{s}=\Delta \dot{x}_{p} . \mathbf{E}_{0} \in R^{4 \times 1}$ is the output disturbance distribution matrix, and $\mathbf{d} \in R^{1}$ is the output disturbance vector.

\section{Observer and Threshold Design}

3.1. Design of Sliding Mode Observers. To realize the fault detection of actuators and sensors, a robust sliding mode observer is designed to make the observer residual insensitive to the change of the disturbance but sensitive to the change of the two types of faults. The design idea of the observer is to take the sensor fault vector and output noise as a part of an augmented state vector and construct a new state space equation with the original state variables. To realize simultaneous multiple faults detection of actuators and sensors, some assumptions are made as follows.
Assumption 1. The nonlinear function vector $\mathbf{g}(\mathbf{x})$ satisfies Lipschitz conditions locally, $\forall \mathbf{x}, \widehat{x} \in R^{n}$

$$
\|\mathbf{g}(\mathbf{x})-\mathbf{g}(\widehat{\mathbf{x}})\| \leq \gamma\|\mathbf{x}-\widehat{\mathbf{x}}\|
$$

where $\widehat{\mathbf{x}}$ is state estimation of $\mathbf{x}$ and $\gamma$ is a positive real Lipschitz constant.

Assumption 2. For every complex number $s$ with nonnegative real part, the rank condition holds:

$$
r\left[\begin{array}{cc}
s I-A & F_{\mathrm{a}} \\
C & 0
\end{array}\right]=n+r\left(F_{\mathrm{a}}\right)
$$

Assumption 3. The matrix $C F_{a}$ is a full column rank as

$$
r\left(C F_{\mathrm{a}}\right)=r\left(F_{\mathrm{a}}\right) .
$$

Remark 1. Assumptions 2 and 3 are necessary and sufficient conditions for the design of a stable sliding mode observer when the system has matched uncertainty or unknown input [13].

Assume there exist nonsingular matrices $R$ and $S$ [16]. Define $\mathbf{z}=R \mathbf{x}=\left[\begin{array}{l}z_{1} \\ z_{2}\end{array}\right]$ and $\mathbf{w}=S \mathbf{y}=\left[\begin{array}{l}w_{1} \\ w_{2}\end{array}\right]$, formula (17) is transformed into

$$
\left\{\begin{array}{l}
\dot{z}=\left[\begin{array}{cc}
A_{1} & A_{2} \\
A_{3} & A_{4}
\end{array}\right] \mathbf{z}+\left[\begin{array}{c}
B_{1} \\
B_{2}
\end{array}\right] u+\left[\begin{array}{c}
R_{1} \\
R_{2}
\end{array}\right] \mathbf{g}\left(R^{-1} \mathbf{z}\right)+\left[\begin{array}{c}
0 \\
F_{\mathrm{a} 2}
\end{array}\right] \mathbf{f}_{\mathrm{a}}+\left[\begin{array}{c}
D_{1} \\
D_{2}
\end{array}\right] F_{\mathrm{d}} \\
\mathbf{w}=\left[\begin{array}{cc}
C_{1} & 0 \\
0 & C_{2}
\end{array}\right] \mathbf{z}+\left[\begin{array}{c}
F_{\mathrm{s} 1} \\
0
\end{array}\right] \mathbf{f}_{\mathrm{s}}+\left[\begin{array}{c}
E_{\mathrm{d} 1} \\
0
\end{array}\right] d
\end{array}\right.
$$

Equation (21) can be rewritten as

$$
\begin{aligned}
& \left\{\begin{array}{l}
\dot{z}_{1}=A_{1} z_{1}+A_{2} z_{2}+B_{1} u+R_{1} g\left(R^{-1} z\right)+D_{1} F_{\mathrm{d}} \\
w_{1}=C_{1} z_{1}+F_{\mathrm{s} 1} f_{\mathrm{s}}+E_{\mathrm{d} 1} d,
\end{array}\right. \\
& \left\{\begin{array}{l}
\dot{z}_{2}=A_{3} z_{1}+A_{4} z_{2}+B_{2} u+R_{2} g\left(R^{-1} z\right)+F_{\mathrm{a} 2} f_{\mathrm{a}}+D_{2} F_{\mathrm{d}}, \\
w_{2}=C_{2} z_{2} .
\end{array}\right.
\end{aligned}
$$

Equations (22) and (23) describe a singular system with stochastic noises.

Construct the augmented system state $\bar{z}$ with the new state vector $\bar{z}_{1}$ and the transformed system state $z_{2}$ as

$$
\bar{z}=\left[\begin{array}{ll}
\bar{z}_{1} & \bar{z}_{2}
\end{array}\right]^{T},
$$

$$
\bar{z}_{1}=\left[\begin{array}{lll}
z_{1} & f_{\mathrm{s}} & d
\end{array}\right]^{T},
$$$$
\bar{z}_{2}=z_{2} \text {, }
$$

$$
\left\{\begin{array}{l}
\bar{E}_{1} \dot{\bar{z}}_{1}=\bar{A}_{1} \bar{z}_{1}+\bar{A}_{2} \bar{z}_{2}+\bar{B}_{1} u+\bar{R}_{1} g\left(R^{-1} \bar{z}\right) \\
\quad+\bar{D}_{1} F_{\mathrm{d}}+N_{\mathrm{s}} f_{\mathrm{s}}+N_{\mathrm{d}} d \\
w_{1}=\bar{C}_{1} \bar{z}_{1},
\end{array}\right.
$$

$$
\left\{\begin{aligned}
\dot{\bar{z}}_{2} & =\bar{A}_{3} \bar{z}_{1}+A_{4} \bar{z}_{2}+B_{2} u+R_{2} g\left(R^{-1} \bar{z}\right) \\
& +F_{\mathrm{a} 2} f_{\mathrm{a}}+D_{2} F_{\mathrm{d}}, \\
w_{2} & =C_{2} \bar{z}_{2},
\end{aligned}\right.
$$


where

$$
\begin{aligned}
& \bar{E}_{1}=\left[\begin{array}{lll}
I & 0 & 0 \\
0 & 0 & 0 \\
0 & 0 & 0
\end{array}\right], \\
& \bar{A}_{1}=\left[\begin{array}{ccc}
A_{1} & 0 & 0 \\
0 & -I & 0 \\
0 & 0 & -I
\end{array}\right] \text {, } \\
& N_{s}=\left[\begin{array}{l}
0 \\
I \\
0
\end{array}\right] \text {, } \\
& \bar{A}_{2}=\left[\begin{array}{c}
A_{2} \\
0 \\
0
\end{array}\right] \text {, } \\
& \bar{C}_{1}=\left[\begin{array}{lll}
C_{1} & F_{\mathrm{s} 1} & E_{\mathrm{d}_{1}}
\end{array}\right], \\
& \bar{B}_{1}=\left[\begin{array}{c}
B_{1} \\
0 \\
0
\end{array}\right], \\
& \bar{A}_{3}=\left[\begin{array}{lll}
A_{3} & 0 & 0
\end{array}\right] \text {, } \\
& \bar{R}_{1}=\left[\begin{array}{c}
R_{1} \\
0 \\
0
\end{array}\right], \\
& \bar{D}_{1}=\left[\begin{array}{c}
D_{1} \\
0 \\
0
\end{array}\right] \text {, } \\
& N_{d}=\left[\begin{array}{l}
0 \\
0 \\
I
\end{array}\right] \text {. }
\end{aligned}
$$

Equations (24) and (25) will be unified as follows:

$$
\left\{\begin{array}{l}
\bar{E} \dot{\bar{z}}=\bar{A} \bar{z}+\bar{B} u+\bar{R} g\left(R^{-1} \bar{z}\right)+\bar{F}_{\mathrm{a}} f_{\mathrm{a}}+\bar{F}_{\mathrm{s}} f_{\mathrm{s}}+\bar{E}_{\mathrm{d}} d+\bar{D} F_{\mathrm{d}}, \\
\bar{w}=\bar{C} \bar{z}
\end{array}\right.
$$

where

$$
\begin{aligned}
& \bar{E}=\left[\begin{array}{ll}
\bar{E}_{1} & 0_{3} \\
0_{3} & I_{3}
\end{array}\right], \\
& \bar{A}=\left[\begin{array}{ll}
\bar{A}_{1} & \bar{A}_{2} \\
\bar{A}_{3} & A_{4}
\end{array}\right],
\end{aligned}
$$

$$
\begin{aligned}
\bar{B} & =\left[\begin{array}{l}
\bar{B}_{1} \\
B_{2}
\end{array}\right], \\
\bar{R} & =\left[\begin{array}{l}
\bar{R}_{1} \\
R_{2}
\end{array}\right], \\
\bar{F}_{\mathrm{a}} & =\left[\begin{array}{l}
0_{3} \\
F_{\mathrm{a} 2}
\end{array}\right], \\
\bar{F}_{\mathrm{s}} & =\left[\begin{array}{l}
N_{\mathrm{s}} \\
0_{3}
\end{array}\right], \\
\bar{E}_{\mathrm{d}} & =\left[\begin{array}{l}
N_{d} \\
0_{3}
\end{array}\right], \\
\bar{D} & =\left[\begin{array}{l}
\bar{D}_{1} \\
D_{2}
\end{array}\right], \\
\bar{C} & =\left[\begin{array}{ll}
\bar{C}_{1} & 0 \\
0 & C_{2}
\end{array}\right] .
\end{aligned}
$$

Equation (27) includes the system states, actuator faults, sensor faults, and output noise.

3.2. Observer Design. To facilitate observer design, the augmented system state $\bar{z}$ can be transformed into $\widetilde{z}$ by $\tilde{z}=\Pi \bar{z}$, where

$$
\begin{aligned}
\prod & =\left[\begin{array}{ll}
\prod_{0} & 0 \\
0 & I
\end{array}\right]=\left[\begin{array}{cc}
\bar{E}_{1}+Q \bar{C}_{1} & 0 \\
0 & I
\end{array}\right], \\
\widetilde{z} & =\left[\begin{array}{c}
\widetilde{z}_{1} \\
\widetilde{z}_{2}
\end{array}\right]=\left[\begin{array}{c}
\prod_{0} \bar{z}_{1} \\
\bar{z}_{2}
\end{array}\right] .
\end{aligned}
$$

For the new state variable $\widetilde{z}$, equations (24) (25) are transformed into

$$
\begin{aligned}
& \left\{\begin{array}{l}
\dot{\tilde{z}}_{1}=\bar{A}_{1} \prod_{0}^{-1} \widetilde{z}_{1}+\bar{A}_{2} \widetilde{z}_{2}+\bar{B}_{1} u+\bar{R}_{1} g\left(R^{-1} E_{0} \Pi^{-1} \widetilde{z}\right) \\
\quad+Q \dot{w}_{1}+\bar{D}_{1} F_{\mathrm{d}}+N_{\mathrm{s}} f_{\mathrm{s}}+N_{\mathrm{d}} d, \\
w_{1}=\bar{C}_{1} \prod_{0}^{-1} \widetilde{z}_{1},
\end{array}\right. \\
& \left\{\begin{array}{c}
\dot{\tilde{z}}_{2}=\bar{A}_{3} \prod_{0}^{-1} \widetilde{z}_{1}+A_{4} \widetilde{z}_{2}+B_{2} u+R_{2} g\left(R^{-1} E_{0} \prod^{-1} \widetilde{z}\right) \\
\quad+F_{\mathrm{a} 2} f_{\mathrm{a}}+D_{2} F_{\mathrm{d}}, \\
w_{2}=C_{2} \widetilde{z}_{2},
\end{array}\right.
\end{aligned}
$$

where $E_{0}=\left[\begin{array}{ll}I & 0\end{array}\right]$. Two sliding mode observers are presented to the system of equations (30) and (31):

$$
\begin{aligned}
& \dot{\widetilde{\widetilde{z}}}_{1}=K_{\mathrm{s}} \widehat{\widetilde{z}}_{1}+\bar{A}_{2} \widehat{\widetilde{z}}_{2}+\bar{B}_{1} u+\bar{R}_{1} g\left(R^{-1} E_{0} \Pi^{-1} \widehat{\widetilde{z}}\right)+Q \dot{w}_{1} \\
& +K_{1} w_{1}-K_{s} Q w_{1}+G_{L 1}\left(w_{2}-C_{2} \widehat{\widetilde{z}}_{2}\right), \\
& \dot{\widetilde{\widetilde{z}}}_{2}=\bar{A}_{3} \prod_{0}^{-1} \widehat{\widetilde{z}}_{1}+A_{4} \widehat{\widetilde{z}}_{2}+B_{2} u+R_{2} g\left(R^{-1} E_{0} \prod^{-1} \widehat{\widetilde{z}}\right) \\
& +G_{L 2}\left(w_{2}-C_{2} \widehat{\widetilde{z}}_{2}\right)+v_{a}
\end{aligned}
$$


where $\widehat{\widetilde{z}}_{1}$ and $\widehat{\widetilde{z}}_{2}$ represent the estimation of $\widetilde{z}_{1}$ and $\widetilde{z}_{2}$ respectively, and $K_{1}$ and $K_{s}$ are unknown matrix. The discontinuous output error injection term $v_{a}$ is defined as

$$
v_{\mathrm{a}}= \begin{cases}\rho_{\mathrm{a}} \frac{P_{2} \widetilde{e}_{2}}{\left\|P_{2} \widetilde{e}_{2}\right\|}, & \widetilde{e}_{2} \neq 0, \\ 0, & \text { others, }\end{cases}
$$

where $\rho_{\mathrm{a}}$ is the observer gain and $P_{2}$ is the symmetric positive definite matrix.

If the state estimation error is defined as $\widetilde{e}=\left[\begin{array}{ll}\widetilde{e}_{1} & \widetilde{e}_{2}\end{array}\right]^{T}$, the dynamics can be obtained as

$$
\begin{aligned}
& \dot{\tilde{e}}_{1}=\dot{\tilde{z}}_{1}-\dot{\widetilde{z}}_{1}=K_{\mathrm{s}} \widetilde{e}_{1}+\left(\bar{A}_{2}-G_{L 1} C_{2}\right) \widetilde{e}_{2}+\bar{D}_{1} F_{\mathrm{d}} \\
& -\left(K_{\mathrm{s}}-\bar{A}_{1} \prod_{0}^{-1}-K_{\mathrm{s}} Q \bar{C}_{1} \prod_{0}^{-1}+K_{1} \bar{C}_{1} \prod_{0}^{-1}\right) \widetilde{z}_{1}+N_{\mathrm{s}} f_{\mathrm{s}} \\
& +\bar{R}_{1}\left[g\left(R^{-1} E_{0} \Pi^{-1} \widetilde{z}\right)-g\left(R^{-1} E_{0} \Pi^{-1} \widetilde{\widetilde{z}}\right)\right]+N_{\mathrm{d}} d, \\
& \dot{\tilde{e}}_{2}=\dot{\tilde{z}}_{2}-\dot{\tilde{\tilde{z}}}_{2}=\bar{A}_{3} \prod_{0}^{-1} \widetilde{e}_{1}+\left(A_{4}-G_{L 2} C_{2}\right) \widetilde{e}_{2} \\
& +R_{2}\left[g\left(R^{-1} E_{0} \Pi^{-1} \widetilde{z}\right)-g\left(R^{-1} E_{0} \Pi^{-1} \widehat{\widetilde{z}}\right)\right]+F_{\mathrm{a} 2} f_{\mathrm{a}} \\
& +D_{2} F_{\mathrm{d}}-v_{\mathrm{a}} \text {. }
\end{aligned}
$$

Equations (32) and (33) can be used as fault detection observers when the following necessary conditions are satisfied:

$$
\begin{aligned}
K_{\mathrm{s}} \bar{E}_{1}+K_{1} \bar{C}_{1} & =\bar{A}_{1}, \\
G_{L 1} & =\bar{A}_{2} C_{2}^{-1}, \\
G_{L 2} & =\left(A_{4}-A_{\mathrm{s} 2}\right) C_{2}^{-1} .
\end{aligned}
$$

Equation (37) holds, and the rank condition of the matrix is satisfied as follows:

$$
r\left(\left[\begin{array}{lll}
\bar{E}_{1} & \bar{C}_{1} & \bar{A}_{1}
\end{array}\right]^{T}\right)=r\left(\left[\begin{array}{ll}
\bar{E}_{1} & \bar{C}_{1}
\end{array}\right]^{T}\right) .
$$

After assigning the parameter values, $r\left(\left[\begin{array}{lll}\bar{E}_{1} & \bar{C}_{1} & \bar{A}_{1}\end{array}\right]^{T}\right)=$ 3 and $r\left(\left[\begin{array}{ll}\bar{E}_{1} & \bar{C}_{1}\end{array}\right]^{T}\right)=3$ are obtained. Thus, the above equation is verified.

The derivative of the error can be simplified as

$$
\begin{aligned}
\dot{\tilde{e}}_{1}= & K_{\mathrm{s}} \widetilde{e}_{1}+\bar{D}_{1} F_{\mathrm{d}}+N_{\mathrm{s}} f_{\mathrm{s}}+N_{\mathrm{d}} d \\
& +\bar{R}_{1}\left[g\left(R^{-1} E_{0} \Pi^{-1} \widetilde{z}\right)-g\left(R^{-1} E_{0} \Pi^{-1} \widehat{\widetilde{z}}\right)\right], \\
\dot{\tilde{e}}_{2}= & A_{\mathrm{s} 2} \widetilde{e}_{2}+\bar{A}_{3} \prod_{0}^{-1} \widetilde{e}_{1}+F_{\mathrm{a} 2} f_{\mathrm{a}}+D_{2} F_{\mathrm{d}}-v_{\mathrm{a}} \\
& +R_{2}\left[g\left(R^{-1} E_{0} \Pi^{-1} \widetilde{z}\right)-g\left(R^{-1} E_{0} \Pi^{-1} \widehat{\widetilde{z}}\right)\right] .
\end{aligned}
$$

Theorem 1. Under necessary conditions equations (37) (39), the error dynamics in equations (41) and (42) approach stable asymptotically, if the inequality

$$
\sum=\left[\begin{array}{ccccc}
\bar{\Gamma}_{1} & \Gamma_{2} & P_{1} \bar{D}_{1} & P_{1} N_{\mathrm{d}} & P_{1} N_{\mathrm{s}} \\
\Gamma_{3} & \bar{\Gamma}_{4} & P_{2} D_{2} & 0 & 0 \\
\bar{D}_{1}^{T} P_{1} & D_{2}^{T} P_{2} & -\sigma^{2} I & 0 & 0 \\
N_{\mathrm{d}}^{T} P_{1} & 0 & 0 & -\sigma^{2} I & 0 \\
N_{\mathrm{s}}^{T} P & 0 & 0 & 0 & -\sigma^{2} I
\end{array}\right]<0,
$$

holds for a symmetric positive definite matrix $P$ and the scalar $\sigma>0$, where $P=\operatorname{diag}\left(P_{1}, P_{2}\right)$,

$$
\begin{aligned}
& \Gamma_{1}=\left(K_{\mathrm{s}}^{T} P_{1}+P_{1} K_{\mathrm{s}}\right)+\kappa \gamma^{2}\left\|R^{-1} E_{0} \prod^{-1}\right\|^{2}+\kappa^{-1} P_{1} \bar{R}_{1}\left(P_{1} \bar{R}_{1}\right)^{T}, \\
& \Gamma_{2}=P_{2} \bar{A}_{3} \prod_{0}^{-1}+\kappa^{-1} P_{1} \bar{R}_{1}\left(P_{2} R_{2}\right)^{T}, \\
& \Gamma_{3}=\left(P_{2} \bar{A}_{3} \prod_{0}^{-1}\right)^{T}+\kappa^{-1} P_{2} R_{2}\left(P_{1} \bar{R}_{1}\right)^{T}, \\
& \Gamma_{4}=\left(A_{\mathrm{s} 2}^{T} P_{2}+P_{2} A_{\mathrm{s} 2}\right)+\kappa \gamma^{2}\left\|R^{-1} E_{0} \Pi^{-1}\right\|^{2}+\kappa^{-1} P_{2} R_{2}\left(P_{2} R_{2}\right)^{T}, \\
& \bar{\Gamma}_{1}=\Gamma_{1}+\bar{C}_{1} \prod_{0}^{-1}\left(\bar{C}_{1} \prod_{0}^{-1}\right)^{T}, \\
& \bar{\Gamma}_{4}=\Gamma_{4}+C_{2} C_{2}^{T} .
\end{aligned}
$$

The proof of this theorem is given in Appendix.

Equation (43) is a linear matrix inequality. According to the Schur complement lemma, the parameter matrices $P$ and $\sigma$ can be solved using the MATLAB LMI toolbox.

Theorem 2 [3]. Under assumptions 1-3 and the observer equations (32) and (33), the trajectories of the error dynamics equations (35) and (36) can be driven to the sliding surface $S=\left\{e_{2} \mid e_{2}=0\right\}$ in finite time if the gain holds as

$$
\rho_{\mathrm{a}} \geq \alpha_{\mathrm{M}}+\left[\left(\left\|\bar{A}_{2} \prod_{0}^{-1}\right\|+\gamma\left\|R_{2}\right\|\left\|R^{-1} E_{0} \Pi^{-1}\right\|\right) \mathcal{E}_{\mathrm{M}}+\left\|D_{1}\right\| \beta_{\mathrm{M}}\right],
$$

where $\varepsilon_{M}$ is the system error upper bound.

Proof. Based on theorem 1, the time derivative of the Lyapunov function $V_{2}=\widetilde{e}_{2}^{\mathrm{T}} P_{2} \widetilde{e}_{2}$ is

$$
\begin{aligned}
\dot{V}_{2}= & \tilde{e}_{2}^{T}\left(A_{\mathrm{s} 2}^{T} P_{2}+P_{2} A_{\mathrm{s} 2}\right) \widetilde{e}_{2}+2 \widetilde{e}_{2}^{T} P_{2} \bar{A}_{3} \prod_{0}^{-1} \widetilde{e}_{1}+2 \widetilde{e}_{2}^{T} P_{2} D_{2} F_{\mathrm{d}} \\
& +2 \widetilde{e}_{2}^{T} P_{2} R_{2}\left[g\left(R^{-1} E_{0} \Pi^{-1} \widetilde{z}\right)-g\left(R^{-1} E_{0} \Pi^{-1} \widehat{\widetilde{z}}\right)\right] \\
& -2 \widetilde{e}_{2}^{T} P_{2} v_{\mathrm{a}}+2 \widetilde{e}_{2}^{T} P_{2} F_{\mathrm{a} 2} f_{\mathrm{a}} .
\end{aligned}
$$

With Cauchy-Schwartz inequalities, $\|\tilde{e}\|=\left\|\widetilde{e}_{1}\right\|+\left\|\widetilde{e}_{2}\right\| \leq \varepsilon_{\mathrm{M}}$, and $A_{\mathrm{s} 2}^{T} P_{2}+P_{2} A_{\mathrm{s} 2}<0$, the time derivative is as follows.

For $\left\|F_{\mathrm{a} 2}\right\|=1$, 


$$
\begin{aligned}
\dot{V}_{2} \leq & 2 \widetilde{e}_{2}^{T} P_{2}\left(\bar{A}_{3} \prod_{0}^{-1} \widetilde{e}_{1}+\gamma R_{2}\left\|R^{-1} E_{0} \Pi^{-1}\right\| \tilde{e}+D_{2} F_{\mathrm{d}}-\alpha_{0 \mathrm{M}}\right) \\
= & 2\left\|\widetilde{e}_{2}^{T} P_{2}\right\|\left(\left\|\bar{A}_{3}\right\|\left\|\prod_{0}^{-1}\right\|\left\|\tilde{e}_{1}\right\|+\gamma\left\|R_{2}\right\|\left\|R^{-1} E_{0} \Pi^{-1}\right\|\|\tilde{e}\|\right) \\
& +\left\|D_{2}\right\|\left\|F_{\mathrm{d}}\right\|-\alpha_{0 \mathrm{M}} \leq-2\left\|\tilde{e}_{2}^{T} P_{2}\right\|\left(\alpha_{0 \mathrm{M}}-\left\|D_{2}\right\| \beta_{\mathrm{M}}\right. \\
& \left.-\left(\left\|\bar{A}_{3} \prod_{0}^{-1}\right\|+\gamma\left\|R_{2}\right\|\left\|R^{-1} E_{0} \Pi^{-1}\right\|\right) \varepsilon_{\mathrm{M}}\right)<0,
\end{aligned}
$$

when

$$
\alpha_{0 \mathrm{M}}-\left[\left(\left\|\bar{A}_{3} \prod_{0}^{-1}\right\|+\gamma\left\|R_{2}\right\|\left\|R^{-1} E_{0} \Pi^{-1}\right\|\right) \varepsilon_{\mathrm{M}}+\left\|D_{2}\right\| \beta_{\mathrm{M}}\right] \geq 0 .
$$

The inequality (45) satisfies

$$
\rho_{\mathrm{a}} \geq \alpha_{\mathrm{M}}+\left[\left(\left\|\bar{A}_{2} \prod_{0}^{-1}\right\|+\gamma\left\|R_{2}\right\|\left\|R^{-1} E_{0} \Pi^{-1}\right\|\right) \varepsilon_{\mathrm{M}}+\left\|D_{1}\right\| \beta_{\mathrm{M}}\right] .
$$

This completes the proof.

3.3. Dynamic Threshold Design. For the ideal state, the observer residual signal should be zero while no fault occurs. However, in actual application, the residual will be approximately to zero, because the state of complete decoupling almost does not exist. The traditional fixed thresholds are less sensitive to faults due to its wide range, especially when incipient faults occur. The dynamic threshold can solve this problem and has the characteristics of a narrow threshold and high sensitivity. Therefore, it is necessary to design a threshold function $\mathbf{J}_{\text {th }}$ to make the residual satisfy

$$
\begin{cases}\mathbf{e} \leq \mathbf{J}_{\text {th }}, & \mathbf{f}_{\mathrm{a}}=0, \\ \mathbf{e}>\mathbf{J}_{\text {th }}, & \mathbf{f}_{\mathrm{a}} \neq 0 .\end{cases}
$$

Velocity residuals are a nonstationary stochastic process, which corresponds to Gauss distribution [4]. The wellknown algorithm, exponentially weighted moving average (EWMA) chart [17], is used to improve the signal-to-noise ratio of velocity residuals:

$$
\mu_{j}=\lambda_{0} r_{j}+\left(1-\lambda_{0}\right) \mu_{j-1}, \quad j=1, \ldots, N,
$$

where $r_{j}$ is the velocity residuals ( $k$ is the sampling number of velocity residuals), and the smoothing factor $\lambda_{0}$ takes as 0.25 .

According to stochastic theories, the mean and variance of velocity residuals are as follows:

$$
\begin{aligned}
& \mu_{r}=\frac{1}{k} \sum_{j=1}^{k} \mu_{j}, \\
& \sigma_{r}^{2}=\frac{1}{k-1} \sum_{j=1}^{k}\left(\mu_{j}-\mu\right)_{r}^{2} .
\end{aligned}
$$

The confidence limit of the mean of velocity residuals is
TAble 1: Parameters of system.

\begin{tabular}{lc}
\hline Parameters & Value \\
\hline$A_{1}\left(\mathrm{~m}^{2}\right)$ & 0.0084 \\
$A_{2}\left(\mathrm{~m}^{2}\right)$ & 0.0084 \\
$A_{3}\left(\mathrm{~m}^{2}\right)$ & 0.0151 \\
$p_{\mathrm{s}}(\mathrm{MPa})$ & 12 \\
$p_{\mathrm{r}}(\mathrm{MPa})$ & 0.15 \\
$V_{01}\left(\mathrm{~m}^{3}\right)$ & 0.0277 \\
$V_{02}\left(\mathrm{~m}^{3}\right)$ & 0.0088 \\
$m_{\mathrm{e}}(\mathrm{kg})$ & 8224.4 \\
$\theta_{0}\left({ }^{\circ}\right)$ & 21.5 \\
$a(\mathrm{~m})$ & 1.075 \\
$b(\mathrm{~m})$ & 2.115 \\
$\tau(\mathrm{s})$ & 0.012 \\
$C_{\mathrm{i}}\left(\mathrm{m}^{3} \cdot \mathrm{s}^{-1} \cdot \mathrm{Pa}^{-1}\right)$ & $5 \times 10^{-15}$ \\
$C_{\mathrm{e}}\left(\mathrm{m}^{3} \cdot \mathrm{s}^{-1} \cdot \mathrm{Pa}^{-1}\right)$ & $1 \times 10^{-15}$ \\
$\beta_{\mathrm{e}}(\mathrm{MPa})$ & 1200 \\
$d_{\mathrm{v}}(\mathrm{m})$ & $5 \times 10^{-5}$ \\
$k_{\mathrm{v}}\left(\mathrm{m}^{-1} \cdot \mathrm{V}^{-1}\right)$ & $1 \times 10^{-3}$ \\
\hline
\end{tabular}

$$
P\left\{\mu_{r}-z \sigma_{r}<\mu_{r}<\mu_{r}+z \sigma_{r}\right\}=1-\alpha,
$$

where $\alpha$ is the confidence level and $z$ is the coefficient. In general engineering practice, $\alpha=0.025$. According to the $z$ test table, the coefficient $z=2.24$.

The dynamic threshold is defined as

$$
\mathbf{J}_{\mathrm{th}}= \begin{cases}\mu_{r} \pm z \sigma_{r}, & \ddot{x}_{\mathrm{p}}>\left|\ddot{x}_{\mathrm{pc}}\right|, \\ v_{0}, & \ddot{x}_{\mathrm{p}} \leq\left|\ddot{x}_{\mathrm{pc}}\right|,\end{cases}
$$

where $v_{0}$ is an empirically stable stage threshold and $\ddot{x}_{p c}$ is the critical acceleration of the hydraulic cylinder. According to equations (50) (55), the fault detection can be realized.

\section{Experimental Results}

To validate the derived model and evaluate the effectiveness of the proposed scheme, the experiment was conducted on a laboratory ESS test rig. The high-performance controller $\mathrm{X} 20 \mathrm{CP} 3585$ of B\&R Industrial Automation Company was employed, and the sampling period was $0.4 \mathrm{~ms}$. The data acquisition module and fault diagnosis system are operated at a rate of $1 \mathrm{kHz}$. Two types of experiments were carried out: (1) the normal state; (2) the fault state.

4.1. Normal State. The slaving process of the elevating servo system of VMH is a typical point-to-point control. To facilitate this study and ensure the representativeness of the experiment, this article considered the upward and downward gun slaving process as a working cycle. Table 1 shows the system parameters.

The sensor output data were considered using white noise signal with mean value 0 and variance value 0.1 . Figure 3 shows the elevating angle of command value, measurement value, and observed value in normal state.

Generally, the hydraulic cylinder undergoes the motion of acceleration-uniform velocity-deceleration process; thereby, the working process can be separated into a stable stage (uniform velocity) and a transitional stage 


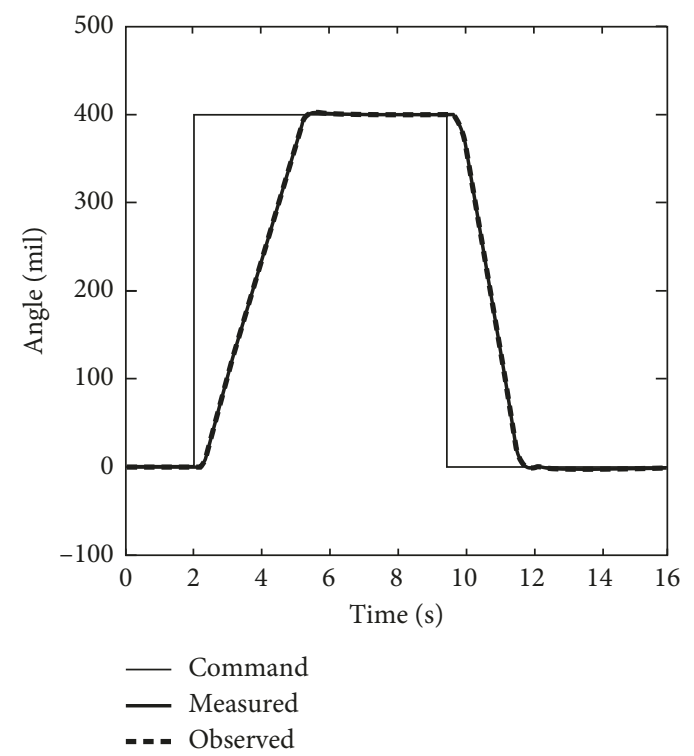

Figure 3: Elevating angle.

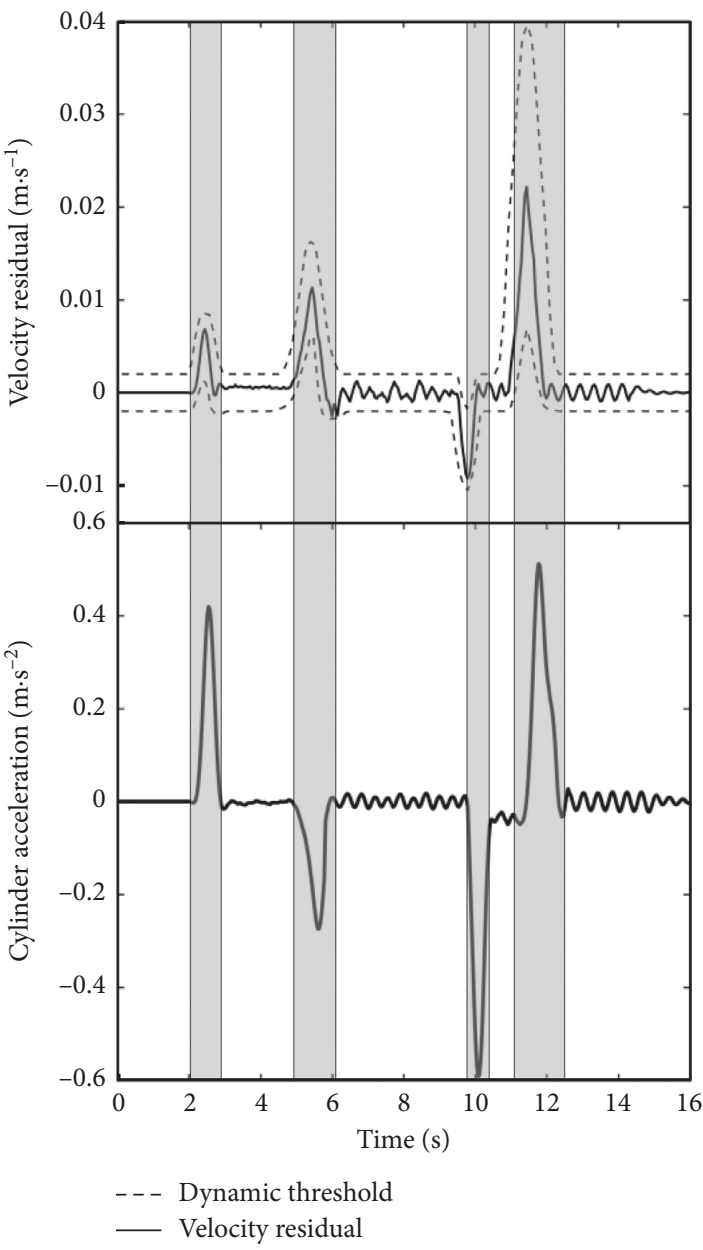

(a)

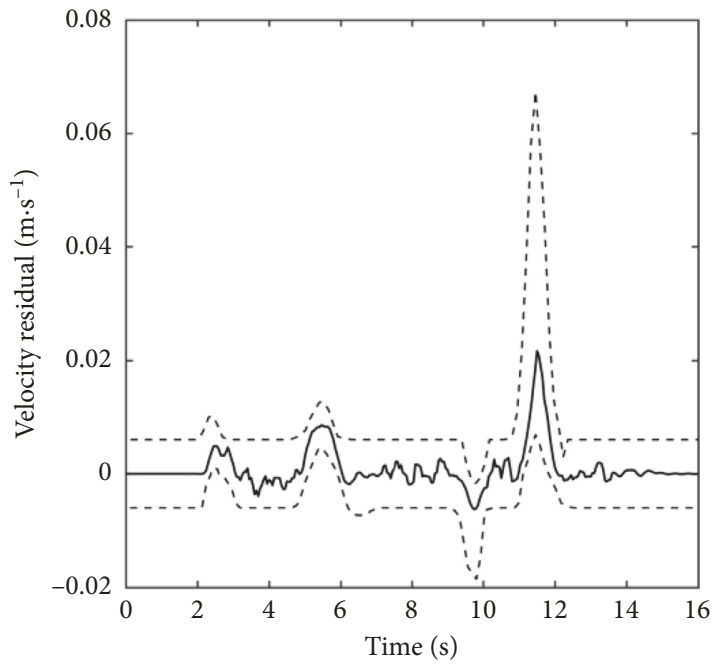

(b)

FIgURE 4: (a) Residuals of cylinder velocity and acceleration in normal state. (b) Residuals of cylinder velocity using method in [18]. 
TABLE 2: Common faults implementation scheme.

\begin{tabular}{lccc}
\hline Serial no. & Faults type & Faults implementation & Parameter setting \\
\hline 1 & System pressure fault & Regulating pressure by a proportional relief valve & $-1.0 \mathrm{MPa}$ \\
2 & Internal leakage & Adding an adjustable throttle valve between cylinder & Throttle diameter 3.5 mm \\
3 & ports & $95 \%$ \\
4 & Proportional fault & Reducing proportional valve amplifier gain & $95 \%$ \\
\hline
\end{tabular}

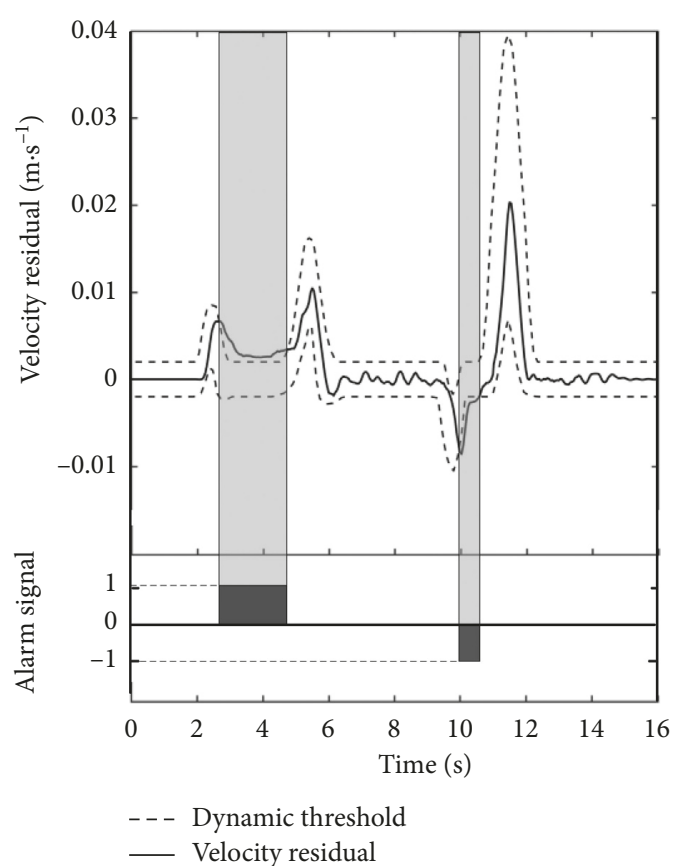

Figure 5: Residuals of system pressure fault and alarm signal.

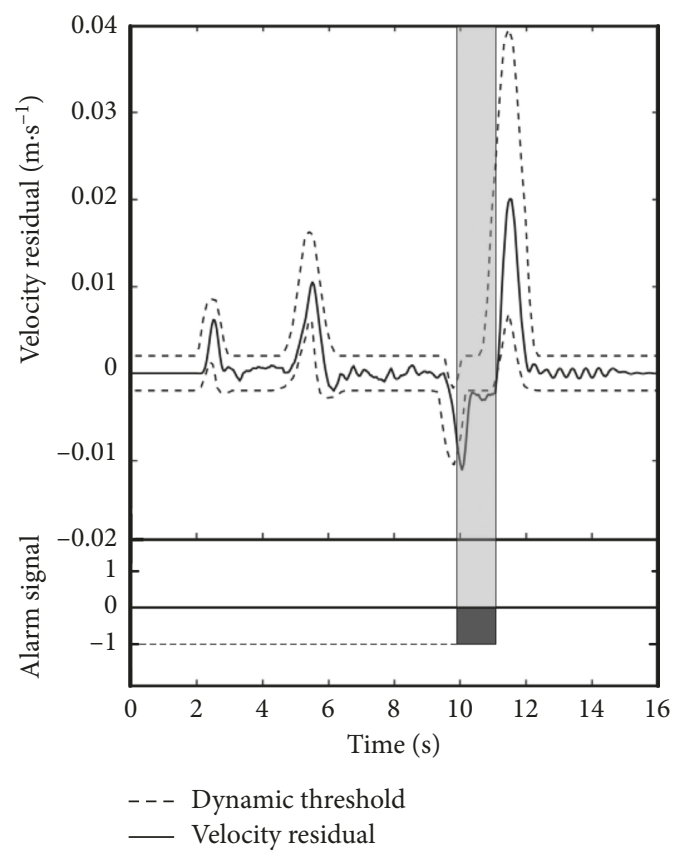

FIGURE 6: Residuals of internal leakage and alarm signal.

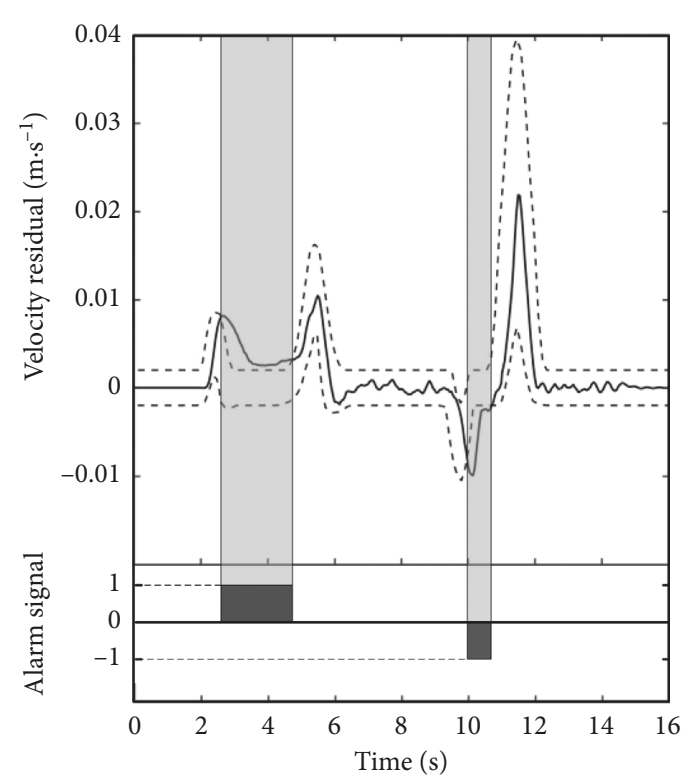

Figure 7: Residuals of proportional fault and alarm signal.

(acceleration or deceleration). Due to the large difference in the acceleration characteristics of the two stages, the instability of the speed residuals signal is shown in Figure 4(a), which was used to distinguish between stable stage and transitional stage. According to several experiments, the critical acceleration of the cylinder was chosen with the value of $\pm 0.002 \mathrm{~m} / \mathrm{s}^{2}$. As shown in Figure 4(a), under the normal working condition, the dotted line shows the upper threshold and lower threshold and solid lines shows the residuals signal. As a comparison, a method based on Wald's sequential test proposed in [18] is applied to the ESS. The result is shown in Figure 4(b). The residuals of cylinder velocity in normal state change in a wide range.

4.2. Fault State. The causes of parameter change in the system were complex and diverse. In order to verify the observer's ability to detect faults, the common faults were set artificially in the laboratory environment based on Table 2.

The failure decision criteria were designed as follows: if the system residual signal exceeds the dynamic threshold, the system could be regarded as faulty, and an alarm signal will be sent out. Figure 5 shows the system state with pressure deviation implemented by substituting the pilot relief valve with a proportional pilot relief valve. Both types of valves have the same pressure-flow rate performance with different control modes only. As seen, the alarm signal 


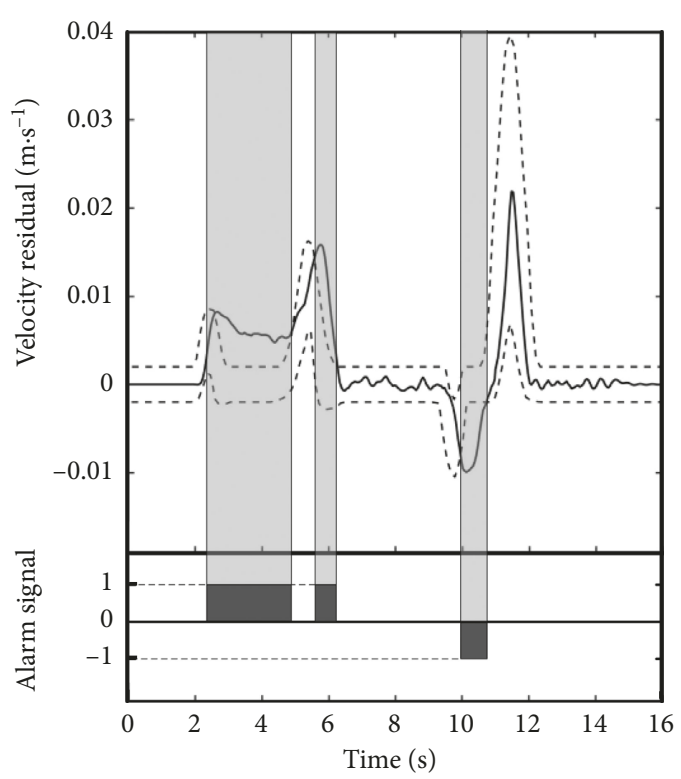

FIgURE 8: Residuals of angular sensor fault and alarm signal.

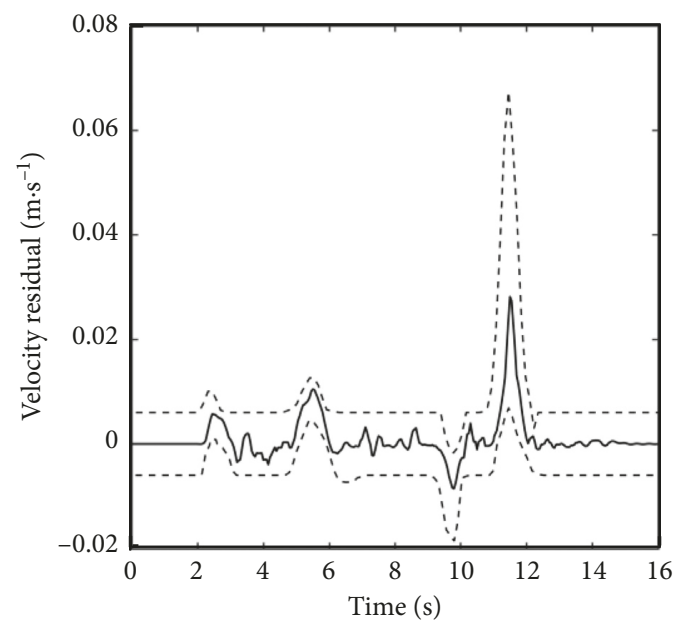

FIGURE 9: Residuals of system pressure fault and alarm signal.

appeared in the whole working cycle. The fault detection system generated some alarm signals when the residuals went beyond the dynamic threshold obviously.

An adjustable throttle valve was added between the extension chamber and retraction chamber with throttle orifice diameter of $3.5 \mathrm{~mm}$ to simulate a state of increasing internal leakage caused by seal wear. Figure 6 shows the fluctuations in residuals exceeding the dynamic threshold with alarm signal.

To conduct the fault state of the flow reduction through the servo valve due to blockage, the maximum displacement of the proportional valve spool was set as $95 \%$ of the normal state. When the spool of the proportional valve is blocked or stuck, the fault detection system could detect this fault and send out an alarm signal. The experimental results are shown in Figure 7.
The angular displacement sensor gain was adjusted to simulate the fault state that the sensing component in the sensor drifts. When the sensor output value deviated, the fault detection system was capable of discerning the fault and producing an alarm signal. The experimental results are shown in Figure 8.

By comparing the methods in reference [18], the system pressure fault is taken as an example to detect the system faults. Because the observer has poor robustness to disturbance, the residual fluctuation is large in the normal state and the threshold must be set wider. As a result, the observer is insensitive to system pressure fault, and the system does not alarm. Compared with Figure 4(b) and Figures 5 and 9, the sliding mode-based observer method shows a better robustness and is more sensitive to faults.

\section{Conclusions}

In the paper, an online fault detection scheme is presented for the ESS with model uncertainty, nonlinearities, unknown disturbance, and output noise, which is capable of improving the safety and reliability of the VMH system. A robust sliding mode-based observer is designed to generate the fault detection residual to detect actuator faults and sensor faults simultaneously. Four experiments on faults are conducted. The correctness of the derived model and some common fault expressions are also validated. A comparative experiment was performed, and the proposed method shows good performance.

Despite the online fault detection scheme was demonstrated in the ESS system, it is expected to be applied to similar industrial systems $[19,20]$.

\section{Appendix}

\section{Proof of Theorem 1}

Consider the Lyapunov function as $V=V_{1}+V_{2}=\widetilde{e}_{1}^{T} P_{1} \widetilde{e}_{1}+$ $\tilde{e}_{2}^{T} P_{2} \widetilde{e}_{2}$, then, its time derivative of $V$ is

$$
\begin{aligned}
\dot{V}= & \dot{V}_{1}+\dot{V}_{2}=\dot{\tilde{e}}_{1}^{T} P_{1} \dot{\tilde{e}}_{1}+\dot{\tilde{e}}_{2}^{T} P_{2} \dot{\tilde{e}}_{2} \\
\dot{V}_{1}= & \widetilde{e}_{1}^{T}\left(K_{\mathrm{s}}^{T} P_{1}+P_{1} K_{\mathrm{s}}\right) \widetilde{e}_{1}+2 \widetilde{e}_{1}^{T} P_{1} \bar{D}_{1} F_{\mathrm{d}}+2 \widetilde{e}_{1}^{T} P_{1} N_{\mathrm{s}} f_{\mathrm{s}} \\
& +2 \widetilde{e}_{1}^{T} P_{1} N_{\mathrm{d}} d+2 \widetilde{e}_{1}^{T} P_{1} \bar{R}_{1}\left[g\left(R^{-1} E_{0} \Pi^{-1} \widetilde{z}\right)\right. \\
& \left.-g\left(R^{-1} E_{0} \Pi^{-1} \widehat{\widetilde{z}}\right)\right] \\
\dot{V}_{2}= & \widetilde{e}_{2}^{T}\left(A_{\mathrm{s} 2}^{T} P_{2}+P_{2} A_{\mathrm{s} 2}\right) \widetilde{e}_{2}+2 \widetilde{e}_{2}^{T} P_{2} \bar{A}_{3} \prod_{0}^{-1} \widetilde{e}_{1} \\
& +2 \widetilde{e}_{2}^{T} P_{2}\left(F_{\mathrm{a} 2} f_{\mathrm{a}}-v_{\mathrm{a}}\right)+2 \widetilde{e}_{2}^{T} P_{2} D_{2} F_{\mathrm{d}} \\
& +2 \widetilde{e}_{2}^{T} P_{2} R_{2}\left[g\left(R^{-1} E_{0} \Pi^{-1} \widetilde{z}\right)-g\left(R^{-1} E_{0} \Pi^{-1} \widehat{\widetilde{z}}\right)\right]
\end{aligned}
$$

Inequality $2 x^{T} y \leq \kappa^{-1} x^{T} x+\kappa y^{T} y$ holds for all $\kappa>0$, $\alpha_{0 M}=\rho_{a}-\left\|F_{\mathrm{a} 2}\right\| \alpha_{M}$. Define 


$$
\begin{aligned}
& \widetilde{V}_{1}=2 \widetilde{e}_{2}^{T} P_{2}\left(F_{\mathrm{a} 2} f_{\mathrm{a}}-v_{\mathrm{a}}\right) \leq-2\left\|\tilde{e}_{2}^{T} P_{2}\right\|\left(\rho_{\mathrm{a}}-\left\|F_{\mathrm{a} 2}\right\| \alpha_{\mathrm{M}}\right) \\
& \leq-2 \alpha_{0 \mathrm{M}}\left\|\tilde{e}_{2}^{T} P_{2}\right\| \leq 0, \\
& \widetilde{V}_{2}=2\left(\widetilde{e}_{1}^{T} P_{1} \bar{R}_{1}+\tilde{e}_{2}^{T} P_{2} R_{2}\right)\left[g\left(R^{-1} E_{0} \Pi^{-1} \widetilde{z}\right)-g\left(R^{-1} E_{0} \Pi^{-1} \widehat{\widetilde{z}}\right)\right] \\
& =2\left[\begin{array}{c}
\widetilde{e}_{1} \\
\tilde{e}_{2}
\end{array}\right]^{T}\left[\begin{array}{cc}
P_{1} & 0 \\
0 & P_{2}
\end{array}\right]\left[\begin{array}{l}
\bar{R}_{1} \\
R_{2}
\end{array}\right]\left(\gamma\left\|R^{-1} E_{0} \Pi^{-1}\right\| I_{2}\right)\left[\begin{array}{c}
\tilde{e}_{1} \\
\widetilde{e}_{2}
\end{array}\right] \\
& \leq\left[\begin{array}{c}
\tilde{e}_{1} \\
\tilde{e}_{2}
\end{array}\right]^{T}\left[\kappa^{-1}\left(\left[\begin{array}{cc}
P_{1} & 0 \\
0 & P_{2}
\end{array}\right]\left[\begin{array}{c}
\bar{R}_{1} \\
R_{2}
\end{array}\right]\right)\left(\left[\begin{array}{cc}
P_{1} & 0 \\
0 & P_{2}
\end{array}\right]\left[\begin{array}{c}
\bar{R}_{1} \\
R_{2}
\end{array}\right]\right)^{T}+\kappa \gamma^{2}\left\|R^{-1} E_{0} \Pi^{-1}\right\|^{2} I_{2}\right]\left[\begin{array}{l}
\tilde{e}_{1} \\
\tilde{e}_{2}
\end{array}\right] \\
& =\left[\begin{array}{c}
\tilde{e}_{1} \\
\tilde{e}_{2}
\end{array}\right]^{T}\left[\begin{array}{cc}
\kappa \gamma^{2}\left\|R^{-1} E_{0} \prod^{-1}\right\|^{2}+\kappa^{-1} P_{1} \bar{R}_{1}\left(P_{1} \bar{R}_{1}\right)^{T} & \kappa^{-1} P_{1} \bar{R}_{1}\left(P_{2} R_{2}\right)^{T} \\
\kappa^{-1} P_{2} R_{2}\left(P_{1} \bar{R}_{1}\right)^{T} & \kappa \gamma^{2}\left\|R^{-1} E_{0} \Pi^{-1}\right\|^{2}+\kappa^{-1} P_{2} R_{2}\left(P_{2} R_{2}\right)^{T}
\end{array}\right]\left[\begin{array}{l}
\tilde{e}_{1} \\
\widetilde{e}_{2}
\end{array}\right], \\
& \widetilde{V}_{3}=\widetilde{e}_{1}^{T}\left(K_{\mathrm{s}}^{T} P_{1}+P_{1} K_{\mathrm{s}}\right) \widetilde{e}_{1}+\widetilde{e}_{2}^{T}\left(A_{\mathrm{s} 2}^{T} P_{2}+P_{2} A_{\mathrm{s} 2}\right) \widetilde{e}_{2}+2 \widetilde{e}_{2}^{T} P_{2} \bar{A}_{3} \prod_{0}^{-1} \widetilde{e}_{1} \\
& =\left[\begin{array}{c}
\widetilde{e}_{1} \\
\widetilde{e}_{2}
\end{array}\right]^{T}\left[\begin{array}{cc}
K_{\mathrm{s}}^{T} P_{1}+P_{1} K_{\mathrm{s}} & P_{2} \bar{A}_{3} \prod_{0}^{-1} \\
\left(P_{2} \bar{A}_{3} \prod_{0}^{-1}\right)^{T} & A_{\mathrm{s} 2}^{T} P_{2}+P_{2} A_{\mathrm{s} 2}
\end{array}\right]\left[\begin{array}{c}
\tilde{e}_{1} \\
\widetilde{e}_{2}
\end{array}\right] \text {, } \\
& \widetilde{V}_{4}=2 \widetilde{e}_{1}^{T} P_{1} \bar{D}_{1} F_{\mathrm{d}}+2 \widetilde{e}_{2}^{T} P_{2} D_{2} F_{\mathrm{d}}+2 \widetilde{e}_{1}^{T} P_{1} N_{\mathrm{d}} d+2 \widetilde{e}_{1}^{T} P_{1} N_{\mathrm{s}} f_{\mathrm{s}} \\
& =\left[\begin{array}{c}
\widetilde{e}_{1} \\
\widetilde{e}_{2}
\end{array}\right]^{T}\left[\begin{array}{cc}
P_{1} & 0 \\
0 & P_{2}
\end{array}\right]\left[\begin{array}{ccc}
\bar{D}_{1} & N_{\mathrm{d}} & N_{\mathrm{s}} \\
D_{2} & 0 & 0
\end{array}\right] \delta+\delta^{T}\left[\begin{array}{ccc}
\bar{D}_{1} & N_{\mathrm{d}} & N_{\mathrm{s}} \\
D_{2} & 0 & 0
\end{array}\right]^{T}\left[\begin{array}{cc}
P_{1} & 0 \\
0 & P_{2}
\end{array}\right]\left[\begin{array}{c}
\widetilde{e}_{1} \\
\widetilde{e}_{2}
\end{array}\right] \\
& =\widetilde{e}^{T} P \Psi \delta+\delta^{T} \Psi^{T} P \widetilde{e},
\end{aligned}
$$

where $\delta=\left[\begin{array}{lll}F_{\mathrm{d}}^{T} & d^{T} & f_{\mathrm{s}}^{T}\end{array}\right]^{T}$ and $\Psi=\left[\begin{array}{ccc}\bar{D}_{1} & N_{\mathrm{d}} & N_{\mathrm{s}} \\ D_{2} & 0 & 0\end{array}\right]$.
The time derivative of $V$ is

$$
\dot{V} \leq \widetilde{V}_{1}+\widetilde{V}_{2}+\widetilde{V}_{3}+\widetilde{V}_{4}=\widetilde{e}^{T} \Gamma \widetilde{e}+\widetilde{e}^{T} P \Psi \delta+\delta^{T} \Psi^{T} P \widetilde{e}
$$

where $\Gamma=\left[\begin{array}{ll}\Gamma_{1} & \Gamma_{2} \\ \Gamma_{3} & \Gamma_{4}\end{array}\right]$.

Define $J=\dot{V}+w^{T} w-\gamma^{2} \delta^{T} \delta$. It satisfies

$$
\begin{aligned}
J & \leq \widetilde{e}^{T} \Gamma \widetilde{e}+\widetilde{e}^{T} P \Psi \delta+\delta^{T} \Psi^{T} P \widetilde{e}+w^{T} w-\gamma^{2} \delta^{T} \delta \\
& =\left[\begin{array}{c}
\widetilde{e} \\
\delta
\end{array}\right]^{T}\left[\begin{array}{ccccc}
\bar{\Gamma}_{1} & \Gamma_{2} & P_{1} \bar{D}_{1} & P_{1} N_{\mathrm{d}} & P_{1} N_{\mathrm{s}} \\
\Gamma_{3} & \bar{\Gamma}_{4} & P_{2} D_{2} & 0 & 0 \\
\bar{D}_{1}^{T} P_{1} & D_{2}^{T} P_{2} & -\sigma^{2} I & 0 & 0 \\
N_{\mathrm{d}}^{T} P_{1} & 0 & 0 & -\sigma^{2} I & 0 \\
N_{\mathrm{s}}^{T} P & 0 & 0 & 0 & -\sigma^{2} I
\end{array}\right]\left[\begin{array}{c}
\widetilde{e} \\
\delta
\end{array}\right] \\
& =m^{T} \sum m,
\end{aligned}
$$

where $m=\left[\begin{array}{ll}\tilde{e} & \delta\end{array}\right]^{T}$.

If inequality (43) holds, then $J<0$. Thus, the error dynamics approach stability asymptotically. The observers are convergent. This completes the proof.

\section{Data Availability}

The data that support the findings of this study are available from the corresponding author upon reasonable request.

\section{Conflicts of Interest}

The authors declare that they have no conflicts of interest.

\section{Acknowledgments}

This work was partially supported by the National Natural Science Foundation of Jiangsu Province of China (Grant no. BK20170816) and the Fundamental Research Funds for the Central Universities (Grant no. 309171B8802).

\section{References}

[1] D. Li and X. Hu, "Redundant and fault-tolerant algorithms for real-time measurement and control systems for weapon equipment," ISA Transactions, vol. 67, pp. 398-406, 2017.

[2] M. Fallahi, M. Zareinejad, K. Baghestan, A. Tivay, S. M. Rezaei, and A. Abdullah, "Precise position control of an electro-hydraulic servo system via robust linear approximation," ISA Transactions, vol. 80, pp. 503-512, 2018.

[3] H. A. Mintsa, R. Venugopal, J. Kenne, and C. Belleau, "Feedback linearization-based position control of an electrohydraulic servo system with supply pressure uncertainty," IEEE Transactions on Control Systems Technology, vol. 20, no. 4, pp. 1092-1099, 2012.

[4] Z. Shi, F. Gu, B. Lennox, and A. D. Ball, "The development of an adaptive threshold for model-based fault detection of a nonlinear electro-hydraulic system," Control Engineering Practice, vol. 13, no. 11, pp. 1357-1367, 2005.

[5] Z.-Y. Wang, C. Lu, and B. Zhou, "Fault diagnosis for rotary machinery with selective ensemble neural networks," 
Mechanical Systems and Signal Processing, vol. 113, pp. 112130, 2018.

[6] L. Wen, X. Li, L. Gao, and Y. Zhang, "A new convolutional neural network-based data-driven fault diagnosis method," IEEE Transactions on Industrial Electronics, vol. 65, no. 7, pp. 5990-5998, 2018.

[7] Y.-S. Sun, Y.-M. Li, G.-C. Zhang, Y.-H. Zhang, and H.-B. Wu, "Actuator fault diagnosis of autonomous underwater vehicle based on improved elman neural network," Journal of Central South University, vol. 23, no. 4, pp. 808-816, 2016.

[8] S. S. Tayarani-Bathaie, Z. N. S. Vanini, and K. Khorasani, "Dynamic neural network-based fault diagnosis of gas turbine engines," Neurocomputing, vol. 125, pp. 153-165, 2014.

[9] J. Li, K. Pan, and Q. Su, "Sensor fault detection and estimation for switched power electronics systems based on sliding mode observer," Applied Mathematics and Computation, vol. 353, pp. 282-294, 2019.

[10] M. Liu and P. Shi, "Sensor fault estimation and tolerant control for Itô stochastic systems with a descriptor sliding mode approach," Automatica, vol. 49, no. 5, pp. 1242-1250, 2013.

[11] Z. Mao, Y. Zhan, G. Tao, B. Jiang, and X.-G. Yan, "Sensor fault detection for rail vehicle suspension systems with disturbances and stochastic noises," IEEE Transactions on Vehicular Technology, vol. 66, no. 6, pp. 4691-4705, 2017.

[12] J. Yang, F. Zhu, X. Wang, and X. Bu, "Robust sliding-mode observer-based sensor fault estimation, actuator fault detection and isolation for uncertain nonlinear systems," International Journal of Control, Automation and Systems, vol. 13, no. 5, pp. 1037-1046, 2015.

[13] J. Zhang, A. K. Swain, and S. K. Nguang, "Simultaneous robust actuator and sensor fault estimation for uncertain non-linear lipschitz systems," IET Control Theory \& Applications, vol. 8, no. 14, pp. 1364-1374, 2014.

[14] J. Yuan, F. Ji, Y. Gao, J. Zhu, C. Wei, and Y. Zhou, "Integrated ensemble noise-reconstructed empirical mode decomposition for mechanical fault detection," Mechanical Systems and Signal Processing, vol. 104, pp. 323-346, 2018.

[15] Z. Gao and H. Wang, "Descriptor observer approaches for multivariable systems with measurement noises and application in fault detection and diagnosis," Systems \& Control Letters, vol. 55, no. 4, pp. 304-313, 2006.

[16] R. Raoufi, H. J. Marquez, and A. S. I. Zinober, " $H_{\infty}$ sliding mode observers for uncertain nonlinear lipschitz systems with fault estimation synthesis," International Journal of Robust and Nonlinear Control, vol. 20, pp. 1785-1801, 2010.

[17] O. Amri, M. Mansouri, A. Al-Khazraji, H. Nounou, M. Nounou, and A. Ben Hamida, "Improved model based fault detection technique and application to humanoid robots," Mechatronics, vol. 53, pp. 140-151, 2018.

[18] H. Khan, S. C. Abou, and N. Sepehri, "Nonlinear observerbased fault detection technique for electro-hydraulic servopositioning systems," Mechatronics, vol. 15, no. 9, pp. 10371059, 2005.

[19] S. Nie, L. Qian, L. Tian, and Q. Zou, "Adaptive sliding mode control for electro-hydraulic position servo system of the elevation-balancing machine of artillery platform," in Proceedings of the IEEE 4th Information Technology and Mechatronics Engineering Conference (ITOEC), pp. 731-735, Chongqing China, December 2018.

[20] Q. Guo, J.-M. Yin, T. Yu, and D. Jiang, "Coupled-disturbanceobserver-based position tracking control for a cascade electrohydraulic system," ISA Transactions, vol. 68, pp. 367-380, 2017. 


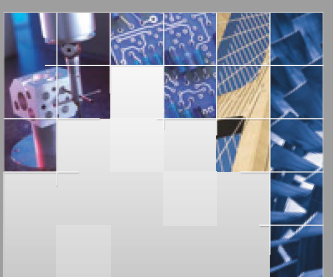

\section{Enfincering}
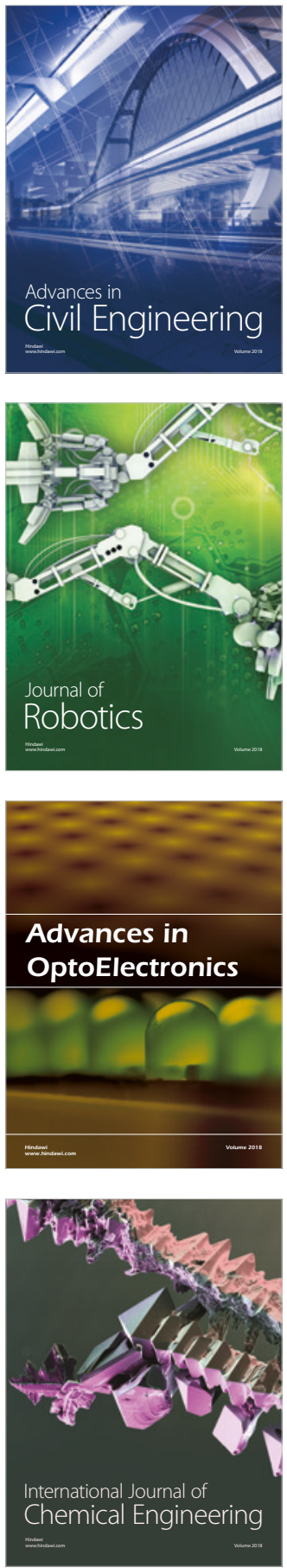

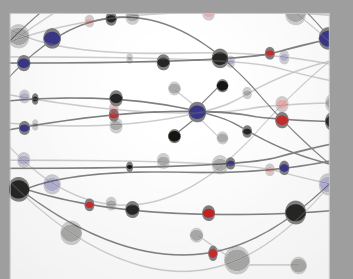

\section{Rotating \\ Machinery}

The Scientific World Journal

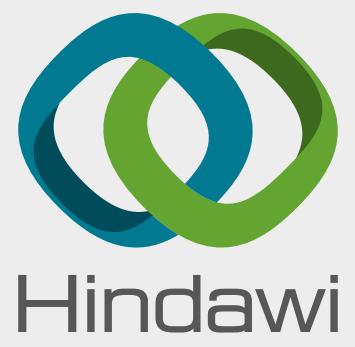

Submit your manuscripts at

www.hindawi.com
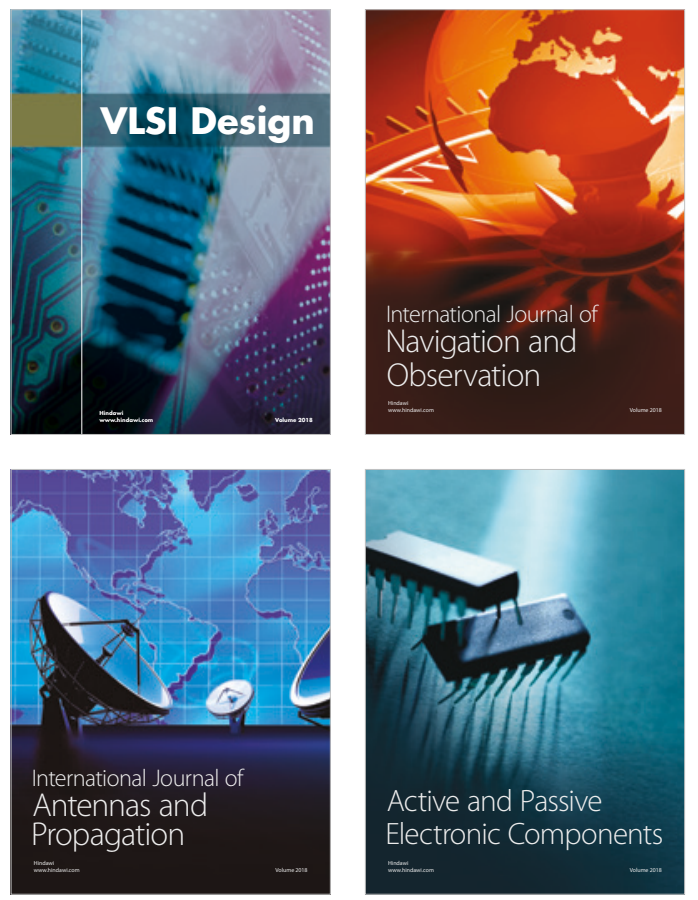
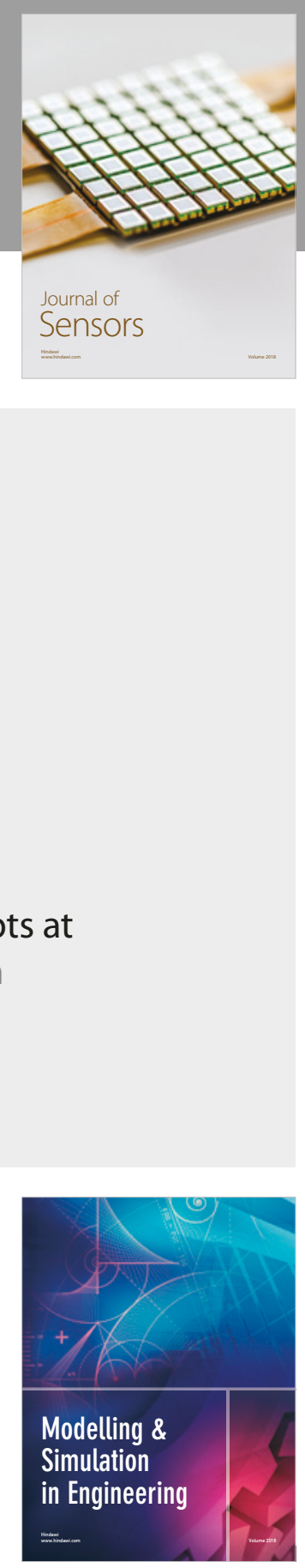

\section{Advances \\ Multimedia}
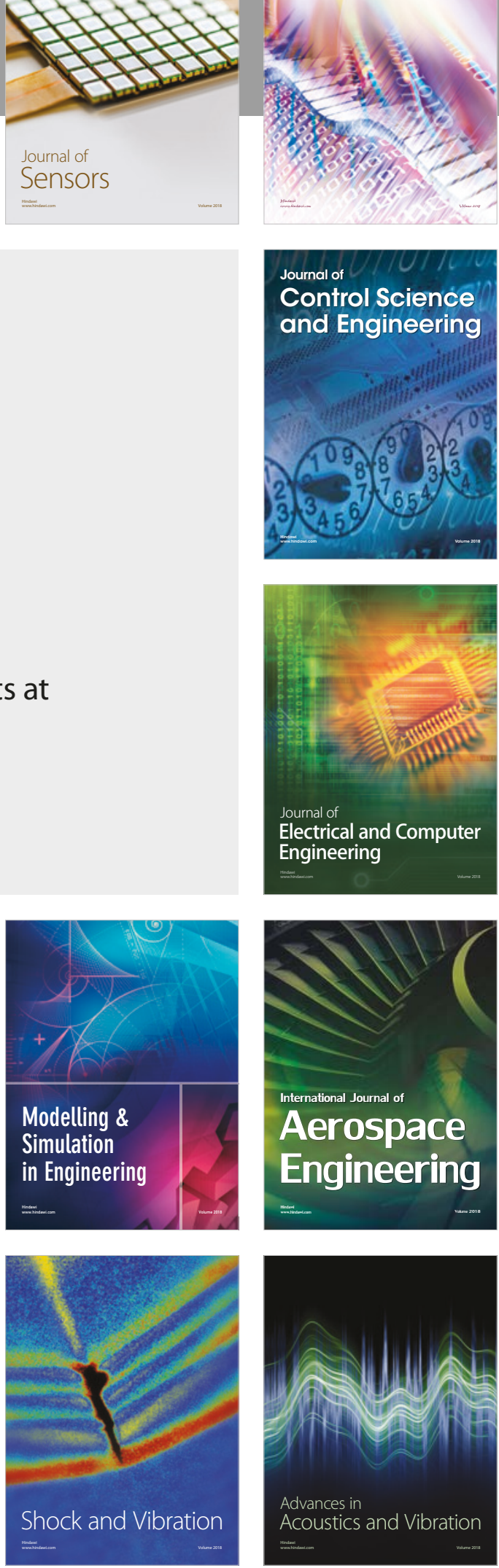